\title{
Lidil
}

Revue de linguistique et de didactique des langues

$64 \mid 2021$

Le passif dans la langue parlée

\section{Catherine Schnedecker, Les chaînes de référence en} français

Paris, Éditions Ophrys, 2021, 276 p.

\section{Francis Grossmann}

\section{(2) OpenEdition}

\section{Journals}

Édition électronique

URL : https://journals.openedition.org/lidil/9353

DOI : $10.4000 /$ lidil.9353

ISSN : 1960-6052

\section{Éditeur}

UGA Éditions/Université Grenoble Alpes

Édition imprimée

ISBN : 978-2-37747-315-1

ISSN : $1146-6480$

\section{Référence électronique}

Francis Grossmann, "Catherine Schnedecker, Les chaînes de référence en français », Lidil [En ligne], 64 | 2021, mis en ligne le 01 novembre 2021, consulté le 24 novembre 2021. URL : http:// journals.openedition.org/lidil/9353; DOI : https://doi.org/10.4000/lidil.9353

Ce document a été généré automatiquement le 24 novembre 2021.

(C) Lidil 


\section{Catherine Schnedecker, Les chaînes de référence en français}

Paris, Éditions Ophrys, 2021, 276 p.

Francis Grossmann

\section{RÉFÉRENCE}

Catherine Schnedecker, Les chaînes de référence en français, Paris, Éditions Ophrys, 2021, $276 \mathrm{p}$.

1 Qu'est-ce qui fait qu'un lecteur est capable de suivre le fil d'une histoire, malgré la multiplication des personnages, des événements, et autres entités référentielles ? Et comment faisons-nous pour lire ou écrire un texte cohérent, dans tout genre de discours, sans perdre celui qui va nous lire ? Y a-t-il des contraintes du même ordre à l'oral ? Catherine Schnedecker, spécialiste reconnue des notions liées aux chaines de référence, livre dans l'excellente collection L'essentiel français dirigée par Catherine Fuchs, un volume de synthèse remarquable et très pédagogique sur ces questions difficiles et complexes.

2 L'ouvrage se compose de six chapitres denses et d'un septième, plus rapide, qui présente quelques pistes et conseils destinés aux apprentis chercheurs s'intéressant à ces questions. Les six premiers chapitres sont accompagnés d'orientations bibliographiques complémentaires, et d'exercices dont les corrigés sont fournis sur le site de l'éditeur, à la page de l'ouvrage. L'organisation d'ensemble permet d'aborder l'ensemble des problématiques liées aux chaines de référence. Sont tout d'abord définies et éclairées (chapitre I) les notions classiques d'anaphore et de coréférence, et celle moins souvent traitée par les grammaires de chaine de référence, dont l'auteur rappelle bien, dans son introduction, les enjeux actuels liés aux travaux en linguistique de corpus et au TAL. Sont ensuite traités (chapitre II) les problèmes plus concrets que soulève l'identification des maillons (ou des mentions) d'une chaine de référence, puis celui de la composition de ces chaines (chapitre III) qui pose de fait la question 
redoutable de la forme et du statut des maillons. L'auteur ne gomme pas la diversité des approches possibles sur ce sujet, d'une conception (comme celle de Charolles) qui accorde l'exclusivité aux expressions référentielles, à une autre plus ouverte à des éléments plus divers pouvant jouer le rôle de « rappel » du référent; préférence semble donnée à une conception intermédiaire, celle de Landragin (2011) consistant à différencier les maillons selon qu'ils s'appuient ou non sur une forme linguistique. Ce chapitre particulièrement important de l'ouvrage permet aussi de faire un bilan des approches cognitives, et d'examiner ensuite, d'un point de vue linguistique, les limites à la liberté du locuteur qu'impose le « suivi référentiel ».

3 Les deux chapitres suivants (IV et V) abordent respectivement les contraintes « externes» pesant sur la composition des chaines de référence (variation selon l'époque, la langue ou famille de langues, le genre discursif, la nature ontologique du référent principal, etc.) et les contraintes «internes" au texte même (avec notamment la question des plans d'organisation textuelle, du découpage paragraphique, titre et sous-titres, discours rapporté, etc.). On peut certes discuter cette présentation entre contrainte externe vs interne, dans la mesure où, par exemple, le genre discursif parait imposer ses contraintes à des unités dites internes comme le découpage paragraphique ou les formes du discours rapporté. Elle permet cependant de mettre en lumière, c'est au moins l'hypothèse formulée à la fin du chapitre $\mathrm{V}$ (p.196), le fait que «les expressions référentielles, loin d'être conditionnées par quelque forme de segmentation que ce soit, découpent à leur manière, le matériau discursif, par leur sémantisme et les instructions interprétatives qu'elles délivrent, en marquant des changements divers (énonciatif, épistémique, conceptuel)». Reste à décrire les formes d'interaction entre ces différents plans de l'organisation textuelle, l'auteur en appelant sur ce point à des expérimentations psycholinguistiques. Le chapitre VI illustre les domaines d'application possibles de la notion de chaine de référence, que cela soit dans le domaine littéraire, ou dans le champ foisonnant actuellement de la linguistique de corpus outillée.

4 Au total, un ouvrage riche au plan théorique, mais abondamment exemplifié, parfois presque un peu trop, l'abondance des textes d'exemples obligeant le lecteur à retrouver ensuite le fil du raisonnement suivi par l'auteur : c'est sans doute là un dernier exercice pratique lié au sujet même qui est traité. L'ouvrage remplit en tous cas parfaitement son rôle d'initiation et deviendra vite indispensable aux cours de sciences du langage.

\section{AUTEURS}

\section{FRANCIS GROSSMANN}

Univ. Grenoble Alpes, LIDILEM, 38000 Grenoble, France 